\section{PARAFFIN PNEUMONIA}

BY

\author{
MICHAEL GELFAND, M.D., M.R.C.P. \\ Physician, Salisbury Native Hospital, Southern Rhodesia
}

It is not uncommon for cases of paraffin (kerosene) poisoning to be admitted to the children's ward of the Salisbury Native Hospital. Since paraffin has many interesting effects, particularly on the respiratory tract, I consider it of general interest to describe the clinical picture that such ingestion might produce. Further recognition of the disorder may easily be overlooked, although doctors practising amongst Africans may be well aware of the consequences of this accident.

The condition is seen particularly among infants living in native compounds, where the paraffin lamp is used for lighting. When the lamp is filled, the remaining paraffin is sometimes not emptied back into the bottle but left in a mug. It is not difficult to appreciate the danger of such a practice, as the crawling infant may easily snatch the container and consume its contents. The child coughs and cries; the mother is alarmed and rushes him to the hospital, where, as a rule, after the stomach is washed out he is admitted for the night.

The infant's condition is usually satisfactory, though there will be some rise in temperature, with the presence of a cough. In most cases the child is not very ill ; but certain physical signs, such as an impairment of the air entry and inspiratory crepitations in the affected parts of the lungs, may be evident. Dullness and tubular breath sounds will not be present unless the patch is large. In most cases the lesions are found in one or both regions of the lower lobes of the lungs.

A radiograph taken at this stage of the illness, about 24 to 36 hours after the onset, generally reveals areas of "cotton-wool" opacities of small irregular size. In the more extensive cases, however, the lesions are lobar or lobular in appearance. They may be unilateral or bilateral.

The fever usually lasts a few days, the temperature then gradually returning to normal. At the height of the fever the respiratory rate may be increased as well as the pulse. The temperature generally varies from 100 to $103^{\circ} \mathrm{F}$. $\left(37.8-39.4^{\circ} \mathrm{C}.\right)$. So far I have not encountered any fatal cases or sequelae; all the patients made an uninterrupted recovery.

The differential diagnosis of paraffin poisoning is that of any acute respiratory affection, such as acute bronchitis or pneumonia. Malaria and acute febrile states may have to be considered also.

\section{Illustrative Cases}

Case 1.-A native male infant aged about 1 year was admitted on January 1, 1949, with a history of having drunk some paraffin the previous. night. The mother did not know what amount had been taken, but believed it was not much. The child coughed and attempted to vomit. He was a well-covered child. The temperature was $99.7^{\circ}$ F. $\left(37.6^{\circ} \mathrm{C}.\right)$; the next evening it had risen to $101^{\circ} \mathrm{F}$. $\left(38.3^{\circ} \mathrm{C}\right.$.), after which it gradually subsided, reaching normal a few days later. On admission the pulse rate was 140 and the respiratory rate 36 a minute, dropping to 130 and 24 respectively after four days. On examination of the chest tubular breathing was audible in the left base, moist crepitations over the same area being heard later. The rest of the physical examination was negative. The white cell count was 13,100 , with $60 \%$ neutrophils, 33\% lymphocytes, and 7\% monocytes. No malaria parasites were found in smears prepared from the peripheral circulation. On admission the stomach was washed out, when the smell of paraffin was detected. No special treatment, such as the use of penicillin or sulphonamides, was given. A radiograph taken on January 26 showed a large area of homogeneous consolidation at the left base, with a more extensive zone of mottled densities in the corresponding area of the right lung.

Case 2.-A native female infant aged about 1 year was admitted on April 25, 1948, with a history of having consumed $2 \mathrm{oz}$. ( $57 \mathrm{ml}$.) of paraffin that day. On admission the temperature was $102^{\circ}$ F. $\left(38.9^{\circ}\right.$ C. $)$ and the child was slightly dyspnoeic. The temperature varied from $100-102^{\circ}$ F. (37.8$38.9^{\circ}$ C.) for the next three days, after which it gradually subsided to normal. Physical examination revealed inspiratory crepitations, mostly at the right base but with a few on the left. The mouth and throat were normal, the abdomen was slightly protuberant, and no enlargement of the spleen or liver was detected. The heart was normal, and no abnormal changes were noted in the nervous system. The urine was clear of albumin and sugar. Peripheral blood smears were negative. A radiograph of the chest taken on April 27 showed a soft area of consolidation in the lower zone of the left lung. On April 29 the child was discharged as fully recovered.

Case 3.-A native male infant aged about 15 months was admitted on December 6, 1948, with a history of having accidentally swallowed $2 \mathrm{oz}$. (57 ml.) of paraffin, after which he had vomited three times. On admission he was coughing, and paraffin could be smelt in the breath. The temperature was $103^{\circ}$ F. $\left(39.4^{\circ} \mathrm{C}\right.$.). He was given a gastric lavage. Examination of the chest revealed crepitations at the left base with bronchial breathing. Nothing else of note was found in the lungs. The mouth, throat, heart, abdomen, and central nervous system all appeared normal. No malaria parasites were found in smears taken of the peripheral blood. The total white cell count was 15,600 (43\% neutrophils, $49 \%$ lymphocytes, $5 \%$ monocytes, and $3 \%$ eosinophils). The patient made a rapid recovery, the temperature returning to normal within three days.

Case 4.-A native female infant aged about 1 year was admitted on February 16, 1949, with a history of having swallowed paraffin a few hours previously. According to her mother, the amount consumed was about $1 \mathrm{oz}$. $(28 \mathrm{ml}$.$) . On$ admission her temperature was $104^{\circ} \mathrm{F}$. $\left(40^{\circ} \mathrm{C}\right.$.), pulse 130 , and respirations 30 . She coughed, but no physical signs were observed in the lungs. A gastric lavage was given soon after admission. A radiograph taken the following day revealed a patchy consolidation in the lower zone of the left lung. The child was given 20,000 units of penicillin three-hourly by intravenous injection and $0.25 \mathrm{~g}$. of sulphadiazine four-hourly by mouth. The temperature subsided rapidly, and two days after admission she had fully recovered and was discharged.

Case 5.-A native female infant aged about 15 months was admitted from the Location Dispensary by Dr. L. Stern, having swallowed paraffin on the afternoon of the previous day. She became very restless and later feverish. The next morning she vomited and developed a cough and some diarrhoea. Later, on admission (February 11, 1949), the temperature was $102^{\circ} \mathrm{F}$. $\left(38.9^{\circ} \mathrm{C}\right.$.) and she was coughing. The mouth and throat were healthy and the abdomen normal. The urine was free of sugar and albumin. The central nervous system was normal. The lungs did not reveal any gross changes on physical examination, but a radiograph showed a well-marked lobar consolidation of the upper zone of the right lung. The stomach was not washed out. The fever gradually subsided to normal after about six days and the patient was discharged.

\section{Discussion}

The literature, especially from America, contains a number of references to paraffin poisoning and pneumonià. Barbour (1926) reported four cases, one of which was fatal. Another fatal case was recorded by Higgins (1933), who suggests that the serious sequelae following the ingestion of paraffin are due to pulmonary involvement from aspiration of the liquid into the lungs. Waring (1933) emphasized the frequency of this type of poisoning. Some of his experiments on dogs tended to show that the pulmonary 
involvement was due to aspiration of the paraffin direct or by regurgitation from the stomach into the lungs. Clinically, $9(39 \%)$ out of 23 patients developed pulmonary changes varying from mild bronchitis to a fatal pneumonia.

Nunn and Martin (1934) recorded their findings in a series of 65 cases. These showed that $43 \%$ of the cases had aspirated some of the paraffin into the lungs. There was a mortality of $9 \%$, all the fatal cases showing evidence of a pneumonitis. Fatalities occurred within the first 18 hours after ingestion. These authors therefore concluded that the prognosis was directly proportional to the amount of hydrocarbon entering the lungs. Their conclusion that all fatalities occurred within 18 hours after the ingestion of paraffin was corroborated by Farabaugh (1936) when he reviewed the various reports on cases of paraffin poisoning found in medical literature.

From experimental work on rabbits, Lesser, Weens, and McKey (1943) concluded that the pulmonary manifestations in these animals were due to aspiration of paraffin into the lungs rather than to its absorption from the gastrointestinal tract. No changes were noted in the lungs of rabbits given varying amounts of paraffin by stomach tube. However, when the paraffin was administered by means of a tracheal catheter, pulmonary oedema and, later, confluent pneumonia were seen. Of a series of 22 cases of paraffin poisoning studied radiographically, $17(77 \%)$ showed pulmonary changes.

Similar experiments on the action of paraffin on the lungs were reported by Deichmann et al. (1944). After administering it to rabbits by mouth or intraperitoneally they concluded that the vascular damage through absorption in the blood stream accounted for changes in the bronchi, and that the pathological lung changes were of the same character whether the paraffin was given by stomach tube or by intraperitoneal injection. In view of lung manifestations due to paraffin carried via the blood stream as well as from direct contact resulting from aspiration, these investigators stressed the necessity for early and efficient removal of all traces of the oil from the gastro-intestinal tract in the emergency treatment. They also showed that the percentage of deaths from the same dose of paraffin varied from nil with highly purified paraffin to as much as 60 with less-purified products.

About the same time Scott (1944) reported what he considered to be the first case in the literature of pneumonia, pneumothorax, and subcutaneous emphysema complicating paraffin poisoning. A similar case was recorded by Lavenstein in 1945.

Steiner (1947) paid particular attention to the clinical picture. He claims that there is an acute intoxication with evidence of cerebral depression, particularly of the vagus and respiratory centres. In most cases recovery takes place in a few days. Many who ingest paraffin develop changes in the lungs, detectable clinically or radiographically. These changes vary from slight infiltration " to more serious complications." Steiner classifies the cases into three groups on the basis of severity and complications: (1) acute toxicity and depression of the central nervous system, with minimal pulmonary changes followed by rapid recovery; (2) severe pneumonia and hyperpyrexia, with prolonged recovery; (3) severe pneumonia, with evidence of degenerative changes in the myocardium, liver, kidneys, and gastro-intestinal tract.

According to Steiner, the central nervous system of infants is sensitive to paraffin. Absorption from the stomach is rapid and likely to produce symptoms within an hour after an accident. These include a rapid and feeble pulse, shallow respirations, cyanosis, and drowsiness, with occasional muscular twitchings. Most infants soon recover from these effects. The lungs, however, are often involved (Group 1). In Group 2 stupor or coma with high fever and more marked changes in the lungs (confluent pneumonia) are seen. In Group 3 the degenerative effects of absorbed paraffin on the liver, spleen, kidneys, and myocardium are superimposed on the toxicity and pneumonia. He describes a case with auricular fibrillation and flutter.

Steiner discusses the two reasons given for the production of the various syndromes-absorption via the blood stream and/or aspiration direct into the bronchi. Clinically, it appears that absorption of paraffin from the stomach has enough pathological potentiality to warrant its prompt removal by gastric lavage. Yet, while such removal has been advocated in the emergency treatment, the development of gastric hyperaemia and occasional multiple erosions of the gastric mucosa necessitate the exercise of caution during this procedure. In evaluating the outlook Steiner stresses the following : (1) age-infants are more susceptible than adults ; (2) amount ingested ; (3) the extent of vomiting and aspiration; and (4) the nature and rapidity of the emergency treatment.

Clinically, it is clearly difficult to state whether the pneumonic changes are due to aspiration of the liquid into the lungs in the first instance or to absorption of the paraffin from the stomach and its eventual settlement in the lungs. In most cases the radiograph reveals basal changes, thus favouring ingestion. It will be noted that in Case 5 of the present series the upper lobe of the right lung only was involved. This might be considered to favour gastric absorption rather than aspiration direct into the lungs.

Steiner claims that the central nervous system of the infant is particularly sensitive to paraffin, but in no case that I have yet seen has there been any undue central nervous depression.

\section{Summary}

Cases of paraffin pneumonia in Southern Rhodesia are reported and the clinical features described.

The prognosis, as a rule, is good, although fatal cases are on record in the literature.

In view of the complications of paraffin poisoning it is advisable to admit all cases to the ward for observation.

My thanks are due to Dr. R. M. Morris, Secretary for Health, Southern Rhodesia, for his permission to publish this paper.

\section{BibliograpHy}

Barbour, O. (1926). J. Amer. med. Ass., 87, 488.

Deichmann, W. B., Kitzmiller, K. V., Witherup, S., and Johansman, R. (1944). Ann. intern. Med., 21, 803.

Far. (1964). Minn. Med. 19, 780.

Fisher, J. H., and Macklin, C. C. (1940). Amer. J. Dis. Child. 60, 102 .

Higgins, J. M. (1933). Penn. Med. J., 36, 526.

Lavenstein, A. F. (1945). J. Pediat., 26, 395.

Lesser L. I., Weens, H. S., and McKey, J. D. (1943). Ibid., 23, 352.

Macklin, C. G. (1937). Canad. med. Ass. J., 36, 414.

Nunn, J. A., and Martin, F. M. (1934). J. Amer. med. Ass., 103, 472 .

Price, J. P. (1932). Ibid., 99, 214.

Scott, E. P. (1944). J. Pediat., 25, 31.

Steiner, M. M. (1947). Amer. J. Dis. Child.. 74, 32.

Waring, J. I. (1933). Amer. J. med. Sci., 185, 325.

A new Health Sciences Building at the University of Washington was formally opened on October 9. It houses the university's schools of medicine, dentistry, and nursing. A college of pharmacy will be built later. The main auditorium, seating 600 people, is wired for television, and when the equipment is complete operations being performed in other parts of the building will be televised. Dr. Edward L. Turner is dean of the medical division. 